\title{
$q$-deformed vibron model for diatomic molecules
}

\author{
R. N. Alvarez, ${ }^{1}$ Dennis Bonatsos, ${ }^{2}$ and Yu. F. Smirnov ${ }^{3, *}$ \\ ${ }^{1}$ Institute of Nuclear Physics, Moscow State University, 117234 Moscow, Russia \\ ${ }^{2}$ Institute of Nuclear Physics, National Center for Scientific Research Demokritos, GR-15310 Aghia Paraskevi, Attiki, Greece \\ ${ }^{3}$ Instituto de Física, Universidad Nacional Autónoma de México, Apartado Postal 20-364, 01000 México, Distrito Federale, Mexico
}

(Received 29 November 1993)

\begin{abstract}
A deformed version of the vibron model for diatomic molecules is constructed. Both the $\mathrm{O}(4)$ and $\mathrm{U}(3)$ dynamical symmetries of the model are rewritten, using the concept of complementary subalgebras, in a more convenient form, which is subsequently deformed. The present model unifies the so far independent successful quantum-algebraic approaches to rotational and to vibrational spectra of diatomic molecules. In addition, the method can be used for the construction of deformed versions of the U(5) and $\mathrm{O}(6)$ limits of the interacting boson model of nuclear structure.
\end{abstract}

PACS number(s): 33.10.Cs, 31.15. +q, 02.20.Sv

\section{INTRODUCTION}

The mathematical structure of quantum algebras (quantum groups) [1-4] has recently been attracting much attention. They are deformed versions of the usual Lie algebras, to which they reduce when the deformation parameter $q$ is set equal to 1 . In parallel, applications of quantum algebras in physics have begun to develop in particular in cases in which Lie algebras are known to describe approximately the symmetries of a physical system. The quantum algebra $\operatorname{su}_{q}(2)$ has been successfully used for describing rotational spectra of diatomic molecules [5-7], deformed nuclei [8-10], and superdeformed nuclei [11]. Vibrational spectra of diatomic molecules have been described in terms of deformed oscillators [12-16], as well as in terms of an $\mathrm{SU}_{q}(1,1)$ symmetry $[17,18]$. Potentials giving spectra equivalent to those of the deformed oscillators just mentioned have been constructed $[19,20]$ and found to be deformed versions of the modified Pöschl-Teller potential or, equivalently, the Morse potential.

On the other hand, the vibron model [21-23], having an overall $\mathrm{U}(4)$ symmetry, is known to provide a unified description of molecular rotations and vibrations through the use of algebraic techniques, in a way similar to the description of collective nuclei in terms of the interacting boson model (IBM) [24]. The O(4) limiting symmetry of the vibron model has been found to be appropriate for diatomic molecules, while the $U(3)$ limiting symmetry has been used for the description of clustering effects in nuclei, as well as for the quasimolecular description of heavy-ion resonances (see [25] for lists of references).

The question is therefore created if a deformed version of the vibron model can accommodate in a unified framework the improved descriptions of rotational and vibra-

\footnotetext{
*On leave of absence from the Institute of Nuclear Physics, Moscow State University, 117234 Moscow, Russia.
}

tional molecular spectra obtained so far in terms of separate quantum algebras. The problem of constructing the deformed version of the vibron model (or of the IBM) is not a simple one since the construction of the reduction chains of $\mathrm{U}_{q}(4)$ and $\mathrm{U}_{q}(6)$ has not been achieved yet. It suffices to be mentioned that the reduction from $\mathrm{SU}_{q}(3)$ to $\mathrm{SO}_{q}(3)$ has been carried out only for fully symmetric irreducible representations (irreps) of $\mathrm{SU}_{q}(3)$ [26]. However, a few efforts towards constructing deformed versions of the vibron model $[27,28]$ and the IBM $[29,30]$ already exist.

In this paper a deformed version of both the $\mathrm{O}(4)$ and $U(3)$ dynamical symmetries of the $U(4)$ vibron model will be constructed, taking advantage of the techniques of complementary algebras, introduced by Quesne and coworkers [31-33], which bypass the difficulties in the construction of reduction chains of quantum algebras. In addition to unifying the existing independent quantumalgebraic descriptions of rotational and of vibrational molecular spectra, the present approach allows, in a simple way, for the introduction of cross terms describing the coupling between these two excitation mechanisms.

A brief account of the vibron model for diatomic molecules will be given in Sec. II, while in Sec. III the model will be formulated in another way, using the techniques of complementary algebras. In Sec. IV the $q$-deformed version of the complementary analogs of both the $\mathrm{O}(4)$ and $U(3)$ dynamical symmetries of the vibron model will be given. Section $V$ will contain discussion of the present results and plans for further work.

\section{THE VIBRON MODEL FOR DIATOMIC MOLECULES}

In this section the briefest possible account of the vibron model [21-23] is given in its usual form. In the vibron model the rotations and vibrations of a diatomic molecule are described in terms of four bosons: a scalar boson of positive parity and angular momentum $l=0$, denoted by $s^{+}$, and the three components of a vector bo- 
son of negative parity and $l=1$, denoted by $p_{\mu}^{+}$, $\mu=0, \pm 1$. The corresponding annihilation operators transforming as spherical tensors are $\tilde{s}=s$ and $\widetilde{p}_{\mu}=(-1)^{1-\mu} p_{-\mu}$. Denoting these bosons by $b_{l, \mu}^{+}, l=0,1$ and $-l \leq \mu \leq l$, and $\widetilde{b}_{l, \mu}=(-1)^{l-\mu} b_{l,-\mu}$, and defining the tensor product of two operators $T_{u_{1}}^{k_{1}}$ and $T_{u_{2}}^{k_{2}}$ as

$\left[T^{k_{1}} \otimes T^{k_{2}}\right]^{k_{3}}=\sum_{u_{1} u_{2}}\left(k_{1} u_{1} k_{2} u_{2} \mid k_{3} u_{3}\right) T_{u_{1}}^{k_{1}} T_{u_{2}}^{k_{2}}$,

one observes that the 16 possible bilinear quantities $\left[b_{l}^{+} \otimes \widetilde{b}_{l^{\prime}}\right]^{L}$ generate the algebra U(4), which is, therefore, the overall symmetry of the vibron model.

There are two chains of subalgebras of $u(4)$ containing the angular-momentum algebra so(3) as a subalgebra. These are

$$
\begin{aligned}
& \text { I: } u(4) \supset o(4) \supset \operatorname{so}(3) \supset \mathrm{so}(2), \\
& \text { II: } u(4) \supset u(3) \supset \operatorname{so}(3) \supset \mathrm{so}(2) \text {. }
\end{aligned}
$$

In the case of chain I the basis has the form $|N \omega L M\rangle$, where the various quantum numbers are defined as follows.

(i) $N$ is the total number of bosons. It characterizes the irreps of $U(4)$, which are fully symmetric, since we are dealing with a system of bosons.

(ii) $\omega$ is the seniority quantum number, characterizing the irreps of $\mathrm{O}(4)$ and obtaining the values $\omega=N, N-2, \ldots, 1$ or 0 .

(iii) $L$ is the angular momentum quantum number, labeling the irreps of $\mathrm{SO}(3)$ and taking the values $L=\omega, \omega-1, \ldots, 1,0$.

(iv) $M$ denotes the $z$ component of the angular momentum, labeling the irreps of $\mathrm{SO}(2)$ and having the values $-L \leq M \leq L$.

When the Hamiltonian is characterized by the dynamical symmetry of chain I, it can be written in terms of the Casimir operators of the algebras appearing in this chain:

$$
\begin{aligned}
H_{\mathrm{I}}= & \epsilon_{0}+\epsilon_{1} C_{1}(\mathrm{u}(4))+\epsilon_{2} C_{2}(\mathrm{u}(4)) \\
& +A C_{2}(\mathrm{o}(4))+B C_{2}(\mathrm{so}(3)),
\end{aligned}
$$

where $N$ and $N^{2}$ are related to the first- and second-order Casimir operators of $u(4)$. The eigenvalues of the Hamiltonian in the basis given above are then

$$
\begin{aligned}
E(N, \omega, L)= & \epsilon_{0}+\epsilon_{1} N+\epsilon_{2} N(N+3) \\
& +A \omega(\omega+2)+B L(L+1) .
\end{aligned}
$$

Usually the vibrational quantum number

$$
v=\frac{N-\omega}{2}
$$

is introduced, and the energy eigenvalues are rewritten as

$$
\begin{aligned}
E(N, v, L)= & \epsilon_{0}^{\prime}+\epsilon_{1}^{\prime} N+\epsilon_{2}^{\prime} N^{2}-4 A(N+2)\left(v+\frac{1}{2}\right) \\
& +4 A\left(v+\frac{1}{2}\right)^{2}+B L(L+1),
\end{aligned}
$$

where $\epsilon_{0}^{\prime}, \epsilon_{1}^{\prime}, \epsilon_{2}^{\prime}$ are related to $\epsilon_{0}, \epsilon_{1}, \epsilon_{2}, A$. It should be noticed that the fourth and fifth terms on the right-hand side correspond to the spectrum of the Morse potential [34].

In the case of chain II the basis is $\left|N n_{p} L M\right\rangle$, where $N$ is again the total number of bosons, while the other quantum numbers are defined as follows.

(i) $n_{p}$ is the number of $p$ bosons labeling the irreps of $\mathrm{U}(3)$ and obtaining the values $n_{p}=0,1, \ldots, N$.

(ii) $L$ is labeling the irreps of $\mathrm{SO}(3)$, obtaining the values $L=n_{p}, n_{p}-2, \ldots, 1$ or 0 .

(iii) $M$ is labeling the irreps of $\mathrm{SO}(2)$, with values $-L \leq M \leq L$.

When the Hamiltonian is characterized by the dynamical symmetry of chain II, it can be written in terms of the Casimir operators of the algebras appearing in it:

$$
\begin{aligned}
H_{\mathrm{II}}= & \epsilon_{0}+\epsilon_{1} C_{1}(\mathrm{u}(4))+\epsilon_{2} C_{2}(\mathrm{u}(4))+\epsilon C_{1}(\mathrm{u}(3)) \\
& +\alpha C_{2}(\mathrm{u}(3))+\beta C_{2}(\mathrm{so}(3)) .
\end{aligned}
$$

The eigenvalues of the Hamiltonian in the basis given above are

$$
\begin{aligned}
E\left(N, n_{p}, L\right)= & \epsilon_{0}+\epsilon_{1} N+\epsilon_{2} N(N+3) \\
& +e n_{p}+\alpha n_{p}\left(n_{p}+2\right)+\beta L(L+1) .
\end{aligned}
$$

\section{ALTERNATIVE FORMULATION OF THE VIBRON MODEL}

An alternative formulation of the vibron model can be achieved in terms of complementary algebras. The notion of complementary algebras was introduced by Moshinsky, Quesne, and co-workers [31-33]. It is especially fruitful in the case of multidimensional harmonic oscillators or many-particle systems of few kinds of bosons. In the present case of four kinds of bosons $\left(s^{+}, p_{\mu}^{+}\right.$, $\mu=0, \pm 1)$ the host algebra is $\operatorname{sp}(8, \mathbb{R})$. Two chains of subalgebras are

$$
\begin{aligned}
& \mathrm{sp}(8, \mathbb{R}) \supset \mathrm{u}(4) \supset \mathrm{o}(4) \supset \mathrm{so}(3) \supset \mathrm{so}(2), \\
& \mathrm{sp}(8, \mathbb{R}) \supset \mathrm{sp}(2, \mathbb{R}) \supset \mathrm{u}(1) .
\end{aligned}
$$

The quantum numbers $N, \omega, L, M$, labeling the irreps of the subalgebras of the first chain, have been described in Sec. II. $\mathrm{sp}(2, \mathbb{R})$ is isomorphic to $\mathrm{su}(1,1)$. The irreps of $\mathrm{su}(1,1)$ and $\mathrm{u}(1)$ are labeled by the quantum numbers $j$ and $m$, respectively. Two subalgebras $A_{1}$ and $A_{2}$ of a larger algebra $A$ are complementary within a definite irrep of $A$ if there is a one-to-one correspondence between all the irreps of $A_{1}$ and of $A_{2}$ contained in this irrep of $A$ [31]. In the example given above, the only irreps of the host algebra $\operatorname{sp}(8, \mathbb{R})$ that can be realized in a Fock boson space are the even irrep [0ं], including the vectors $|N \omega L M\rangle$ with $N$ even, and the odd irrep [i] ], including the vectors with $N$ odd. It can then be proved that o(4) and $\operatorname{sp}(2, \mathbb{R})$ [and thus also o(4) and $\mathrm{su}(1,1)$ ] are complementary. The same holds for $u(4)$ and $u(1)$.

For convenience let us denote the four kinds of bosons introduced in Sec. II by $b_{v}^{\dagger}, v=1,2,3,4$, corresponding to $p_{+1}^{\dagger}, p_{-1}^{\dagger}, p_{0}^{\dagger}, s^{\dagger}$, respectively. To each kind $v$ of bosons corresponds an algebra $\operatorname{sp}^{v}(2, \mathbb{R})$, generated by

$$
K_{+}^{v}=\frac{1}{2} b_{v}^{\dagger} b_{v}^{\dagger}, \quad K_{-}^{v}=\frac{1}{2} b_{v} b_{v}, \quad K_{0}^{v}=\frac{1}{2}\left(N_{v}+\frac{1}{2}\right),
$$


where $N_{v}=b_{v}^{\dagger} b_{v}$. These generators satisfy the commutation relations

$$
\left[K_{0}^{v}, K_{ \pm}^{v}\right]= \pm K_{ \pm}^{v},\left[K_{+}^{v}, K_{-}^{v}\right]=-2 K_{0}^{v} .
$$

The $\operatorname{sp}(2, \mathbb{R}) \approx \operatorname{su}(1,1)$ algebra, mentioned above, is realized in the space of four kinds of bosons. Therefore we are going to use for it the symbol $\operatorname{sp}^{(1234)}(2, \mathbb{R}) \approx \operatorname{su}^{(1234)}(1,1)$. This algebra is generated by

$$
K_{+}=\frac{1}{2} \sum_{v} b_{v}^{\dagger} b_{v}^{\dagger}, \quad K_{-}=\frac{1}{2} \sum_{v} b_{v} b_{v}, \quad K_{0}=\frac{1}{2}(N+2),
$$

where $N=\sum_{v} b_{v}^{\dagger} b_{v}$. These generators satisfy the commutation relations

$$
\left[K_{0}, K_{ \pm}\right]= \pm K_{ \pm}, \quad\left[K_{+}, K_{-}\right]=-2 K_{0} .
$$

The Casimir operator is

$$
C_{2}\left(\operatorname{sp}^{(1234)}(2, \mathbb{R})\right)=-K_{+} K_{-}+K_{0}\left(K_{0}-1\right),
$$

with eigenvalue $j(j+1)$. It is known that when $o(n)$ and su $(1,1)$ are complementary, the quantum numbers $\omega$ and $j$ characterizing their irreps are connected by [35]

$$
j=\frac{1}{2}\left[\omega+\frac{n-4}{2}\right) \text {. }
$$

In the present case $\operatorname{su}^{(1234)}(1,1)$ is complementary to o(4), so that

$$
j=\frac{\omega}{2}=\frac{N-v}{4} .
$$

It is also known that the Casimir operators of two algebras complementary to each other are connected by a simple, usually linear, relation of the type

$$
C_{2}\left(A_{1}\right)=c_{1} C_{2}\left(A_{2}\right)+c_{2} \text {. }
$$

In the case of $o(n)$ and su(1,1) this relation is

$$
C_{2}(\operatorname{su}(1,1))=\frac{1}{4} C_{2}(o(n))+\frac{n(n-4)}{16},
$$

which in the present case of $o(4)$ reduces to

$$
C_{2}\left(\mathrm{su}^{(1234)}(1,1)\right)=\frac{1}{4} C_{2}(\mathrm{o}(4)) \text {. }
$$

The $u(1)$ subalgebra of $s u^{(1234)}(1,1)$ is generated by the operator $K_{0}$ alone, the eigenvalues of which we label by $m$. In the general case of the complementary algebras $\mathrm{u}(n)$ and $\mathrm{u}(1)$, the quantum numbers $N$ and $m$ characterizing their irreps are connected by [35]

$$
m=\frac{1}{2}\left(N+\frac{n}{2}\right),
$$

which in the present special case of $u(4)$ and $u(1)$ reduces to

$$
m=\frac{1}{2}(N+2) \text {. }
$$

The chain of Eq. (3.1) already studied is of interest in the case of the chain I of the vibron model. It implies that in studying chain $I$, one can replace in the basis
$|N \omega L M\rangle$ the quantum numbers $N \omega$ by the quantum numbers $\mathrm{jm}$ of the complementary subalgebras. Furthermore, in the Hamiltonian of Eq. (2.4) one is entitled to replace the second-order Casimir operator of o(4) by the second-order Casimir operator of $\operatorname{su}^{(1234)}(1,1)$ and the first- and second-order Casimir operators of $\mathrm{u}(4)[N$ and $N(N+3)]$ by the first- and second-order Casimir operators of $\mathrm{u}(1)\left(K_{0}\right.$ and $\left.K_{0}^{2}\right)$.

In the case of chain II, the $u(3)$ subalgebra of $u(4)$ involves only the $p$ bosons. The host algebra is then $\operatorname{sp}(6, \mathbb{R})$, having the two chains of subalgebras

$$
\begin{aligned}
& \operatorname{sp}(6, \mathbb{R}) \supset \mathrm{u}(3) \supset \mathrm{so}(3) \supset \mathrm{so}(2), \\
& \mathrm{sp}(6, \mathbb{R}) \supset \mathrm{su}^{(123)}(1,1) \supset \mathrm{u}(1),
\end{aligned}
$$

where the superscript (123) means that only the bosons $b_{1}^{\dagger}, b_{2}^{\dagger}, b_{3}^{\dagger}$ are involved in the formation of $\operatorname{su}^{(123)}(1,1) \approx \operatorname{sp}^{(123)}(2, \mathbb{R})$. Further details on these chains are given below [see Eqs. (3.26) - (3.28)].

The building up of the bases related to the two limiting symmetries of the vibron model can then be achieved as follows. To each kind of boson $b_{v}^{\dagger}$, an $\operatorname{sp}^{v}(2, \mathbb{R})$ algebra corresponds, as already mentioned, generated by the operators given in Eq. (3.3). The states with $N_{v}$ even correspond to the irrep $D^{-3 / 4}$, while the states with $N_{v}$ odd correspond to the irrep $D^{-1 / 4}$. Thus to each boson state

$$
\begin{aligned}
\left|N_{1} N_{2} N_{3} N_{4}\right\rangle= & \frac{1}{\sqrt{N_{1} ! N_{2} ! N_{3} ! N_{4} !}} \\
& \times\left(b_{1}^{\dagger}\right)^{N_{1}}\left(b_{2}^{\dagger}\right)^{N_{2}}\left(b_{3}^{\dagger}\right)^{N_{3}}\left(b_{4}^{\dagger}\right)^{N_{4}}|0\rangle
\end{aligned}
$$

one can correspond a set of four noncompact "angular momenta" $j_{v}=-\frac{3}{4}$ or $-\frac{1}{4}, v=1,2,3,4$, the value of each angular momentum $j_{v}$ depending on the parity of the corresponding boson number $N_{v}$.

We can now proceed to the vector coupling of the first two angular momenta $j_{1}$ and $j_{2}$, using the $\mathrm{SU}(1,1)$ Clebsch-Gordan coefficients $[36,37]$

$$
\begin{gathered}
\left|j_{1} j_{2}: j_{12} m_{12}\right\rangle=\sum_{m_{1} m_{2}}\left\langle j_{1} m_{1} j_{2} m_{2} \mid j_{12} m_{12}\right\rangle_{\mathrm{SU}(1,1)} \\
\times\left|j_{1} m_{1}\right\rangle\left|j_{2} m_{2}\right\rangle .
\end{gathered}
$$

This means that the intermediate $\operatorname{su}^{(12)}(1,1)$ algebra has been introduced, generated by

$$
K_{\mu}^{12}=K_{\mu}^{1}+K_{\mu}^{2}
$$

with $\mu=0, \pm 1$. The host algebra of this space of two kinds of bosons is $\operatorname{sp}(4, \mathbb{R})$. The following two chains of subalgebras exist:

$$
\begin{aligned}
& \operatorname{sp}(4, \mathbb{R}) \supset \mathrm{u}(2) \supset \mathrm{so}(2), \\
& \mathrm{sp}(4, \mathbb{R}) \supset \mathrm{su}^{(12)}(1,1) \supset \mathrm{u}(1),
\end{aligned}
$$

where the irreps of $u(2)$ are labeled by the total number of bosons $N_{12}=N_{1}+N_{2}$, while the irreps of so(2) are labeled by $M=N_{1}-N_{2}$. $\mathrm{SO}(2) \quad$ is complementary to $\operatorname{su}^{(12)}(1,1) \approx \operatorname{sp}^{(12)}(2, \mathbb{R})$, the irreps of which are labeled by

$$
j_{12}=\frac{1}{2}(M-1) \text {, }
$$


according to Eq. (3.8), while $\mathrm{u}(2)$ is complementary to $u(1)$, the irreps of which are labeled by

$$
m_{12}=\frac{1}{2}\left(N_{12}+1\right),
$$

according to Eq. (3.13).

The next step along this line is to couple $j_{12}$ with $j_{3}$. In this case three kinds of bosons are involved, so that the host algebra is $\operatorname{sp}(6, \mathbb{R})$. The relevant chains for this case have been given in Eqs. (3.15) and (3.16), so(3) being complementary to $\mathrm{su}^{(123)}(1,1)$, which is generated by

$$
K_{\mu}^{123}=K_{\mu}^{12}+K_{\mu}^{3},
$$

with $\mu=0, \pm 1$. The resulting eigenvectors are

$$
\begin{aligned}
& \left|j_{1} j_{2}\left(j_{12}\right) j_{3}: j_{123} m_{123}\right\rangle \\
& =\sum_{m_{12} m_{3}}\left\langle j_{12} m_{12} j_{3} m_{3} \mid j_{123} m_{123}\right\rangle_{\mathrm{SU}(1,1)} \\
& \quad \times\left|j_{1} j_{2}: j_{12} m_{12}\right\rangle\left|j_{3} m_{3}\right\rangle
\end{aligned}
$$

The Casimir operators of $\operatorname{so}(3)$ and $\operatorname{su}^{(123)}(1,1)$ are connected by

$$
C_{2}(\mathrm{so}(3))=4 C_{2}\left(\mathrm{su}^{(123)}(1,1)\right)+\frac{3}{4},
$$

according to Eq. (3.11), while the quantum numbers labeling their irreps, $L$ and $j_{123}$, respectively, are connected by

$$
j_{123}=\frac{1}{2}\left(L-\frac{1}{2}\right) \text {, }
$$

according to Eq. (3.8). The eigenvalues of $C_{2}\left(\operatorname{su}^{(123)}(1,1)\right)$ in the above-mentioned basis are given by $j_{123}\left(j_{123}+1\right)$. Furthermore, in the chains of Eqs. (3.15) and (3.16), $u(3)$ and $u(1)$ are complementary, the quantum numbers $n_{p}$ and $m_{123}$ labeling, respectively, their irreps being connected by

$$
m_{123}=\frac{1}{2}\left(n_{p}+\frac{3}{2}\right) \text {, }
$$

according to Eq. (3.13).

The coupling of the two angular momenta $j_{1}$ and $j_{2}$, performed above, can be avoided by noticing that the $\mathrm{su}^{(12)}(1,1)$ can be generated by

$K_{+}^{12}=b_{1}^{\dagger} b_{2}^{\dagger}, \quad K_{-}^{12}=b_{1} b_{2}, \quad K_{0}^{12}=\frac{1}{2}\left(N_{1}+N_{2}+1\right)$.

The vectors

$$
\left|j_{12} m_{12}\right\rangle=\frac{1}{\sqrt{N_{1} ! N_{2} !}}\left(b_{1}^{\dagger}\right)^{N_{1}}\left(b_{2}^{\dagger}\right)^{N_{2}}|0\rangle,
$$

with

$$
j_{12}=\frac{1}{2}\left(N_{1}-N_{2}-1\right), \quad m_{12}=\frac{1}{2}\left(N_{1}+N_{2}+1\right),
$$

[which are in agreement with Eqs. (3.22) and (3.23)] are eigenvectors of the Casimir operator $C_{2}\left(\mathrm{su}^{(12)}(1,1)\right)$, with eigenvalues $j_{12}\left(j_{12}+1\right)$. In this way one can avoid the coupling of $j_{1}$ and $j_{2}$ to $j_{12}$. The coupling of $j_{12}$ and $j_{3}$ cannot be avoided however. The resulting vectors in this case we denote by $\left|j_{12} j_{3}: j_{123} m_{123}\right\rangle$.

In the last step the coupling of $j_{123}$ to $j_{4}$ is performed.
The host algebra in this case is $s p(8, \mathbb{R})$, the relevant chains having been given in Eqs. (3.31) and (3.32). The basis vectors, denoted by $\left|j_{1} j_{2}\left(j_{12}\right) j_{3}\left(j_{123}\right) j_{4}: j m\right\rangle$, or by $\left|j_{12} j_{3}\left(j_{123}\right) j_{4}: j m\right\rangle$ in the case in which the shortened version of Eq. (3.30) is used for the vectors with angular momentum $j_{12}$, correspond to the irreps of the $\operatorname{sp}^{1234}(2, \mathbb{R}) \approx \operatorname{su}^{1234}(1,1)$ algebra, generated by

$$
K_{\mu}^{1234}=K_{\mu}^{123}+K_{\mu}^{4}
$$

with $\mu=0, \pm 1$. Here $K_{\mu}^{4}$ are the generators of the $\mathrm{su}^{4}(1,1)$ algebra, associated with the $s$-bosons. The total noncompact angular momentum $j$, characterizing the irreps of $\mathrm{su}^{(1234)}(1,1)$, is connected to the seniority quantum number $\omega$, characterizing the irreps of o(4), by Eq. (3.8), while the Casimir operators of these two complementary algebras are connected by Eq. (3.12).

Given the above, it is clear that for the chain I of the vibron model, instead of the basis $|N \omega L M\rangle$, the basis $\left|j_{12} j_{3}\left(j_{123}\right) j_{4}: j m\right\rangle$ can be used. Furthermore, in the case of chain II, instead of the basis $\left|N n_{p} L M\right\rangle$, the basis $\left|j_{12} j_{3}: j_{123} m_{123}\right\rangle\left|j_{4} m_{4}\right\rangle$ can be used. It is clear that the connection between the two new bases for the dynamical symmetries of the vibron model is

$$
\begin{aligned}
& \left|j_{12} j_{3}\left(j_{123}\right) j_{4}: j m\right\rangle \\
& =\sum_{m_{123} m_{4}}\left\langle j_{123} m_{123} j_{4} m_{4} \mid j m\right\rangle_{\mathrm{SU}(1,1)} \\
& \quad \times\left|j_{12} j_{3}: j_{123} m_{123}\right\rangle\left|j_{4} m_{4}\right\rangle
\end{aligned}
$$

The Hamiltonian of chain I, given in Eq. (2.4), can be rewritten using the complementarity relations described in Eqs. (3.1), (3.2), (3.15), and (3.16), as

$$
\begin{aligned}
H_{\mathrm{I}}= & \epsilon_{0}^{\prime}+\epsilon_{1}^{\prime} C_{1}(\mathrm{u}(1))+\epsilon_{2}^{\prime} C_{2}(\mathrm{u}(1)) \\
& +4 A C_{2}\left(\mathrm{su}^{(1234)}(1,1)\right)+4 B C_{2}\left(\mathrm{su}^{(123)}(1,1)\right) .
\end{aligned}
$$

The eigenvalues of this Hamiltonian are

$$
\begin{aligned}
E\left(m, j, j_{123}\right)= & \epsilon_{0}^{\prime}+\epsilon_{1}^{\prime} m+\epsilon_{2}^{\prime} m^{2}+4 A j(j+1) \\
& +4 B j_{123}\left(j_{123}+1\right) .
\end{aligned}
$$

Using Eqs. (3.14), (3.9), and (3.27), which connect the quantum numbers $m, j, j_{123}$ to the previous ones $(N, \omega, L)$, it is easily verified that Eq. (3.35) is an alternative way of writing Eq. (2.5).

Similarly the Hamiltonian of chain II, given in Eq. (2.8), can be rewritten, taking into account the complementarity relations given in Eqs. (3.1), (3.2), (3.15), and (3.16), as

$$
\begin{aligned}
H_{\mathrm{II}}= & \epsilon_{0}^{\prime}+\epsilon_{1}^{\prime} m+\epsilon_{2}^{\prime} m^{2}+\epsilon^{\prime} m_{123}+\alpha^{\prime} m_{123}^{2} \\
& +4 \beta^{\prime} C_{2}\left(\mathrm{su}^{(123)}(1,1)\right),
\end{aligned}
$$

where on the right-hand side the second and third term correspond to the first- and second-order Casimir operator of the $u(1)$ algebra of the chain of Eq. (3.2), while the fourth and fifth terms correspond to the first- and second-order Casimir operators of the $u(1)$ algebra appearing in the chain of Eq. (3.16). The eigenvalues of this 
Hamiltonian are

$$
\begin{aligned}
E\left(m, m_{123}, j_{123}\right)= & \epsilon_{0}^{\prime}+\epsilon_{1}^{\prime} m+\epsilon_{2}^{\prime} m^{2}+\epsilon^{\prime} m_{123}+\alpha^{\prime} m_{123}^{2} \\
& +4 \beta^{\prime} j_{123}\left(j_{123}+1\right) .
\end{aligned}
$$

Using Eqs. (3.14), (3.28), and (3.27), which connect $m, m_{123}, j_{123}$ to $N, n_{p}, L$, it is easily verified that Eq. (3.37) is an alternative way of writing Eq. (2.9).

In this section we have therefore rewritten the bases and the Hamiltonians corresponding to the two dynamical symmetries of the vibron model in terms of complementary subalgebras. This formulation is useful because it can be $q$ deformed in a very simple way.

\section{IV. $q$ DEFORMATION OF THE VIBRON MODEL}

In the preceding section the subalgebra chains of the vibron model were reduced to equivalent chains of complementary subalgebras

$$
\mathrm{su}^{(1234)}(1,1) \supset \mathrm{su}^{(123)}(1,1) \supset \mathrm{su} \mathrm{u}^{(12)}(1,1) \supset \mathrm{u}(1) .
$$

The corresponding Hamiltonians were then written in terms of the Casimir operators of the new reduction chains. An evident possibility for $q$ deforming these Hamiltonians is to substitute the su(1,1) algebras of Eq. (4.1) by their $q$-deformed counterparts $\mathrm{su}_{q}(1,1)$ [38-40],

$$
\operatorname{su}_{q}^{(1234)}(1,1) \supset \operatorname{su}_{q}^{(123)}(1,1) \supset \operatorname{su}_{q}^{(12)}(1,1) \supset \mathrm{U}_{q}(1) \text {. }
$$

In this section we shall explain how this can be achieved, after giving a brief account of the necessary mathematical details.

$q$ numbers are defined as

$$
[x]_{q}=\frac{q^{x}-q^{-x}}{q-q^{-1}} .
$$

For $q$ real ( $q=e^{\tau}$ with $\tau$ real), they can be written as

$$
[x]_{q}=\frac{\sinh \tau x}{\sinh \tau}
$$

while in the case of $q$ being a phase ( $q=e^{i \tau}$ with $\tau$ real), they obtain the form

$$
[x]_{q}=\frac{\sin \tau x}{\sin \tau} .
$$

In the limiting $q \rightarrow 1(\tau \rightarrow 0), q$ numbers reduce to usual numbers.

$q$-deformed oscillators $[41,42]$ are introduced through the relations

$a a^{\dagger}-q^{ \pm 1} a^{\dagger} a=q^{\mp N}, \quad\left[N, a^{\dagger}\right]=a^{\dagger}, \quad[N, a]=-a$,

where $a^{\dagger}$ and $a$ are the $q$-deformed boson creation and annihilation operators and $N$ the relevant number operator. Using Eq. (4.6) one can easily show that

$$
a^{\dagger} a=[N]_{q}, \quad a a^{\dagger}=[N+1]_{q} .
$$

$q$-deformed algebras can be expressed in terms of $q$ deformed bosons. Introducing $a_{i}^{\dagger}$ as the $q$-deformed analogs of $b_{i}^{\dagger}(i=1,2,3,4)$, with the properties

$$
\left[a_{v}^{\dagger}, a_{\mu}^{\dagger}\right]=\left[a_{v}, a_{\mu}\right]=\left[a_{v}, a_{\mu}^{\dagger}\right]=0, \quad v \neq \mu,
$$

one can prove that $\operatorname{su}_{q}^{(12)}(1,1)$ is generated by [38-40]

$$
K_{+}^{(12)}=a_{1}^{\dagger} a_{2}^{\dagger}, \quad K_{-}^{(12)}=a_{1} a_{2}, \quad K_{0}^{(12)}=\frac{1}{2}\left(N_{1}+N_{2}+1\right),
$$

the relevant commutation relations being

$$
\begin{aligned}
& {\left[K_{0}^{(12)}, K_{ \pm}^{(12)}\right]= \pm K_{ \pm}^{(12)},} \\
& {\left[K_{+}^{(12)}, K_{-}^{(12)}\right]=-\left[2 K_{0}^{(12)}\right]_{q} .}
\end{aligned}
$$

The vectors

$\left|j_{12} m_{12}\right\rangle_{q}=\frac{1}{\sqrt{\left[N_{1}\right]_{q} !\left[N_{2}\right]_{q} !}}\left(a_{1}^{\dagger}\right)^{N_{1}}\left(a_{2}^{\dagger}\right)^{N_{2}}|0\rangle$,

with $j_{12}, m_{12}$ still given by Eq. (3.31), are eigenvectors of the deformed Casimir operator

$C_{2}\left(\operatorname{su}_{q}^{(12)}(1,1)\right)=-K_{+}^{(12)} K_{-}^{(12)}+\left[K_{0}^{(12)}\right]_{q}\left[K_{0}^{(12)}-1\right]_{q}$,

with eigenvalues $\left[j_{12}\right]_{q}\left[j_{12}+1\right]_{q}$.

For the $\operatorname{su}^{v}(1,1)$ algebras one has the boson realization $[38]$

$$
K_{+}^{v}=\frac{1}{[2]_{q}} a^{\dagger} a^{\dagger}, \quad K_{-}^{v}=\frac{1}{[2]_{q}} a a, \quad K_{0}^{v}=\frac{1}{2}\left(N_{v}+\frac{1}{2}\right)
$$

where $N_{v}$ is the number of $v$ bosons. These generators satisfy the commutation relations

$$
\left[K_{0}^{v}, K_{ \pm}^{v}\right]= \pm K_{ \pm}^{v}, \quad\left[K_{+}^{v}, K_{-}^{v}\right]=-\left[2 K_{0}^{v}\right]_{q^{2}} .
$$

We are not going to use the $\operatorname{su}_{q}^{1}(1,1)$ and $\operatorname{su}_{q}^{2}(1,1)$ algebras explicitly in couplings, since for the $\operatorname{su}_{q}^{\left(12^{2}\right)}(1,1)$ algebra we already have the form given in Eq. (4.9), which avoids the direct coupling. In order to be able to couple $\operatorname{su}_{q}^{3}(1,1)$ and $\operatorname{su}_{q}^{4}(1,1)$ to $\operatorname{su}_{q}^{(12)}(1,1)$, it is useful to have the same deformation parameter in all of these algebras, i.e., it is useful to have the same deformation parameter in the commutation relations of Eqs. (4.10) and (4.14). In order to achieve that, we replace in Eqs. (4.13) and (4.14) $q^{2}$ by $q$. As a result, for $v=3,4$, Eq. (4.6) is meant from now on with $q$ replaced by $\sqrt{q}$. Then one also has

$$
a_{v}^{\dagger} a_{v}=\left[N_{v}\right]_{\sqrt{q}}, \quad a_{v} a_{v}^{\dagger}=\left[N_{v}+1\right]_{\sqrt{q}} .
$$

Equation (4.12), giving the Casimir operator, is therefore valid in this case with the usual $q$ numbers.

The $\operatorname{su}_{q}^{(123)}(1,1)$ algebra is generated by the operators

$$
\begin{aligned}
& K_{ \pm}^{(123)}=K_{ \pm}^{(12)} q^{K_{0}^{3}}+K_{ \pm}^{3} q^{-K_{0}^{(12)}}, \\
& K_{0}^{(123)}=K_{0}^{(12)}+K_{0}^{3}
\end{aligned}
$$

i.e., it is a standard coproduct of the irreps $D^{j_{12}+}$ and $D^{j_{3}+}$ of the $\operatorname{su}_{q}(1,1)$ algebra. Therefore the basis vectors, in analogy to Eq. (3.25), are of the form 


$$
\begin{aligned}
& \left|j_{12} j_{3}: j_{123} m_{123}\right\rangle_{q} \\
& =\sum_{m_{12} m_{3}}\left\langle j_{12} m_{12} j_{3} m_{3} \mid j_{123} m_{123}\right\rangle_{\mathrm{SU}_{q}(1,1)} \\
& \quad \times\left|j_{12} m_{12}\right\rangle_{q}\left|j_{3} m_{3}\right\rangle_{q},
\end{aligned}
$$

where $\left\langle j_{1} m_{1} j_{2} m_{2} \mid j m\right\rangle_{\mathrm{SU}_{q}(1,1)}$ are Clebsch-Gordan coefficients for the tensor product of two $\operatorname{su}_{q}(1,1)$ irreps. Explicit analytical formulas for these coefficients, as well as for the relevant $\mathrm{su}_{q}(2)$ coefficients, can be found in [43-47].

The $\operatorname{su}_{q}^{(1234)}(1,1)$ algebra is generated by the operators

$$
K_{ \pm}^{(1234)}=K_{ \pm}^{(123)} q^{K_{0}^{4}}+K_{ \pm}^{4} q^{-K_{0}^{(123)}}
$$

while the vectors analogous to Eq. (3.33) are

$$
\begin{aligned}
& \left|j_{12} j_{3}\left(j_{123}\right) j_{4}: j m\right\rangle_{q} \\
& =\sum_{m_{123} m_{4}}\left\langle j_{123} m_{123} j_{4} m_{4} \mid j m\right\rangle_{\mathrm{SU}_{q}(1,1)} \\
& \quad \times\left|j_{12} j_{3}: j_{123} m_{123}\right\rangle_{q}\left|j_{4} m_{4}\right\rangle_{q} .
\end{aligned}
$$

These vectors are the $q$ analogs of the eigenvectors of the dynamical symmetry I of the vibron model. Similarly the vectors

$$
\left|j_{12} j_{3}: j_{123} m_{123}\right\rangle_{q}\left|j_{4} m_{4}\right\rangle_{q}
$$

are the $q$ analogs of the eigenvectors of the dynamical symmetry II of the vibron model. Therefore Eq. (4.19) connects the eigenvectors of the two dynamical symmetries, as Eq. (3.33) does in the classical case.

In the case of dynamical symmetry I the Hamiltonian reads

$$
\begin{aligned}
H_{\mathrm{I}}= & \epsilon_{0}+\epsilon_{1}[m]_{q}+\epsilon_{2}[m]_{q}^{2}+4 A C_{2}\left(\mathrm{su}_{q}^{(1234)}(1,1)\right) \\
& +4 B C_{2}\left(\mathrm{su}_{q}^{(123)}(1,1)\right),
\end{aligned}
$$

which is the $q$ analog of Eq. (3.34) (with the primes of the coefficients dropped). The eigenvalues of this Hamiltonian are

$$
\begin{aligned}
E\left(m, j, j_{123}\right)= & \epsilon_{0}+\epsilon_{1}[m]_{q}+\epsilon_{2}[m]_{q}^{2}+4 A[j]_{q}[j+1]_{q} \\
& +4 B\left[j_{123}\right]_{q}\left[j_{123}+1\right]_{q} .
\end{aligned}
$$

In the limit $q \rightarrow 1$, Eq. (3.35) is obtained. Assuming that $m, j, j_{123}$ are still connected to quantum numbers $N, \omega, L$ through Eqs. (3.14), (3.9), and (3.27), Eq. (4.22) can be rewritten in a way resembling its classical counterpart, Eq. (2.5), as

$$
\begin{aligned}
E(N, \omega, L)= & \epsilon_{0}^{\prime}+\epsilon_{1}^{\prime}[N+2]_{\sqrt{q}}+\epsilon_{2}^{\prime}[N+2]_{\sqrt{q}}^{2} \\
& +A^{\prime}[\omega]_{\sqrt{q}}[\omega+2]_{\sqrt{q}}+B^{\prime}[L]_{\sqrt{q}}[L+1]_{\sqrt{q}} .
\end{aligned}
$$

In producing Eq. (4.23), identities such as

$$
\begin{aligned}
& {\left[\frac{\omega}{2}\right]_{q}=[\omega]_{\sqrt{q}}\left(q^{1 / 2}+q^{-1 / 2}\right)^{-1},} \\
& {\left[L-\frac{1}{2}\right]_{q}\left[L+\frac{3}{2}\right]_{q}=[L]_{q}[L+1]_{q}-\left[\frac{1}{2}\right]_{q}\left[\frac{3}{2}\right]_{q}}
\end{aligned}
$$

have been used. Using Eq. (2.6), Eq. (4.23) can be written as

$$
\begin{aligned}
E(N, v, L)= & \epsilon_{0}^{\prime}+\epsilon_{1}^{\prime}[N+2]_{\sqrt{q}}+\epsilon_{2}^{\prime}[N+2]_{\sqrt{q}}^{2} \\
& +A\left[v-\frac{N}{2}\right]_{q}\left[v-1-\frac{N}{2}\right]_{q} \\
& +B^{\prime}[L]_{\sqrt{q}}[L+1]_{\sqrt{q}},
\end{aligned}
$$

which reduces to Eq. (2.7) in the limit $q \rightarrow 1$, up to a redefinition of $\epsilon_{0}^{\prime}, \epsilon_{1}^{\prime}, \epsilon_{2}^{\prime}$.

In the case of the dynamical symmetry II the Hamiltonian can be written as

$$
\begin{aligned}
H_{\mathrm{II}}= & \epsilon_{0}+\epsilon_{1}[m]_{q}+\epsilon_{2}[m]_{q}^{2}+\epsilon\left[m_{123}\right]_{q} \\
& +\alpha\left[m_{123}\right]_{q}^{2}+\beta C_{2}\left(\mathrm{su}_{q}^{(123)}(1,1)\right),
\end{aligned}
$$

which is the $q$ analog of Eq. (3.36) (with the primes of the coefficients dropped). The eigenvalues of this Hamiltonian are

$$
\begin{aligned}
E\left(m, m_{123}, L\right)= & \epsilon_{0}+\epsilon_{1}[m]_{q}+\epsilon_{2}[m]_{q}^{2}+\epsilon\left[m_{123}\right]_{q} \\
& +\alpha\left[m_{123}\right]_{q}^{2}+\beta\left[j_{123}\right]_{q}\left[j_{123}+1\right]_{q} .
\end{aligned}
$$

In the limit $q \rightarrow 1$, Eq. (3.37) is obtained. Assuming that $m, m_{123}, j_{123}$ are connected to $N, n_{p}, L$ through Eqs. (3.14), (3.28), and (3.27), Eq. (4.28) can be rewritten in a way resembling its classical counterpart, Eq. (2.9), as

$$
\begin{aligned}
E\left(N, n_{p}, L\right)= & \epsilon_{0}^{\prime}+\epsilon_{1}^{\prime}[N+2]_{\sqrt{q}}+\epsilon_{2}^{\prime}[N+2]_{\sqrt{q}}^{2} \\
& +\epsilon^{\prime}\left[n_{p}+\frac{3}{2}\right]_{\sqrt{q}}+\alpha^{\prime}\left[n_{p}+\frac{3}{2}\right]_{\sqrt{q}}^{2} \\
& +\beta^{\prime}[L]_{\sqrt{q}}[L+1]_{\sqrt{q}} .
\end{aligned}
$$

The results obtained in this section call for the following comments.

(i) Rotational-vibrational spectra of diatomic molecules are described empirically by the Dunham expansion [48]

$$
E(v, L)=\sum_{i k} Y_{i k}\left(v+\frac{1}{2}\right)^{i}[L(L+1)]^{k},
$$

where $v$ is the vibrational quantum number, $L$ the angular momentum, and $Y_{i k}$ the Dunham coefficients, fitted to experiment. It is clear that the Dunham expansion contains powers of $\left(v+\frac{1}{2}\right)$, powers of $L(L+1)$, as well as cross terms. Equations (4.23) and (4.29) contain no cross terms. This is due to the fact that in the Hamiltonians of Eqs. (4.21) and (4.27), only terms up to quadratic in the generators are included, as in the case of the classical vibron model. Cross terms can be taken into account in the dynamical symmetry I, for example, by modifying Eq. (4.21) as follows:

$H_{\mathrm{I}}^{\prime}=H_{\mathrm{I}}+D C_{2}\left(\mathrm{su}_{q}^{(1234)}(1,1)\right) C_{2}\left(\mathrm{su}_{q}^{(123)}(1,1)\right)$.

Then Eq. (4.26) is modified as

$$
\begin{gathered}
E^{\prime}(N, v, L)=E(N, v, L)+D^{\prime}\left[v-\frac{N}{2}\right]_{q}\left[v-1-\frac{N}{2}\right]_{q} \\
\times[L]_{\sqrt{q}}[L+1]_{\sqrt{q}} .
\end{gathered}
$$


(ii) Rotational spectra in both dynamical symmetries [Eqs. (4.26) and (4.29)] are described by the term $[L]_{\sqrt{q}}[L+1]_{\sqrt{q}}$. This is known to be the Casimir operator of $\mathrm{su}_{\sqrt{q}}(2)$. The $\mathrm{su}_{q}(2)$ model has been extensively used for the description of rotational spectra of di- atomic molecules [5-7] and deformed [8-10] and superdeformed [11] nuclei. It has been found [9] that this term is equivalent to an expansion in terms of powers of $L(L+1)$,

$$
\begin{gathered}
{[L]_{q}[L+1]_{q}=\frac{1}{\left\{j_{0}(\tau)\right\}^{2}}\left(j_{0}(\tau) L(L+1)-\tau j_{1}(\tau)\{L(L+1)\}^{2}+\frac{2}{3} \tau^{2} j_{2}(\tau)\{L(L+1)\}^{3}\right.} \\
\left.-\frac{1}{3} \tau^{3} j_{3}(\tau)\{L(L+1)\}^{4}+\frac{2}{15} \tau^{4} j_{4}(\tau)\{L(L+1)\}^{5}-\cdots\right)
\end{gathered}
$$

where $j_{n}(\tau)$ are the spherical Bessel functions of the first kind and $q=e^{i \tau}$. This expansion is similar to the one contained in the Dunham expansion. In the case of $\mathrm{su}_{q}(2)$, however, all the expansion coefficients are related to powers of $\tau$, thus resulting in economy of parameters. Notice that the decreasing of the coefficients of increasing powers of $L(L+1)$, as well as the alternating signs of the terms, facts that are known empirically to hold, occur in Eq. (4.33) automatically, since $\tau$ is known [5-11] to obtain small positive values. Furthermore, it has been proved [9] that the $\mathrm{su}_{q}(2)$ model is equivalent to the vari- able moment of inertia (VMI) model, which describes rotational stretching effects. The $q$ parameter has been found [9] to correspond to the softness parameter of the VMI model. The implications of the $\operatorname{su}_{q}(2)$ model on the electromagnetic transition probabilities connecting the rotational levels of nuclei have been considered [10].

(iii) The fourth term in Eq. (4.26) corresponds to the Casimir operator of $\operatorname{su}_{q}(1,1)$, already used [17] for the description of vibrational spectra of diatomic molecules. It has been proved [17] that this term, for $q=e^{i \tau}$, can be expanded as

$$
\begin{aligned}
{\left[v-\frac{N}{2}\right]_{q}\left[v-1-\frac{N}{2}\right]_{q}=\frac{1}{\sin (\tau)^{2}} } & \frac{1}{2}(\cos (\tau)-\cos \{\tau(N+2)\})-\tau \sin \{\tau(N+2)\}\left(v+\frac{1}{2}\right) \\
& +\tau^{2} \cos \{\tau(N+2)\}\left(v+\frac{1}{2}\right)^{2}+\frac{2}{3} \tau^{3} \sin \{\tau(N+2)\}\left(v+\frac{1}{2}\right)^{3} \\
& \left.-\frac{1}{3} \tau^{4} \cos \{\tau(N+2)\}\left(v+\frac{1}{2}\right)^{4}+\cdots\right\} .
\end{aligned}
$$

We remark that a series of powers of $\left(v+\frac{1}{2}\right)$ is obtained, similar to the one contained in the Dunham expansion. In the present case, however, the expansion coefficients are all related to $\tau$ (and $N$, which in the vibron model is a constant for a given molecule), thus resulting in economy in parameters.

(iv) The anharmonicity constant (i.e., the ratio $\left.Y_{20} / Y_{10}\right)$ in the classical case [Eq. (2.7)] is fixed to $-1 /(N+2)$. In the deformed case of Eq. (4.26), however, it is equal to $-\tau / \tan [\tau(N+2)]$, as it is easily seen from the expansion of Eq. (4.34). The extra freedom gained this way has been found [17] to improve the fits of vibrational molecular spectra.

(v) Since $N$ is fixed for a given molecule (related to the maximum number of bound states below the dissociation limit), the first three terms in Eqs. (4.26) and (4.29) have no influence on the spectrum.

(vi) In Eq. (4.26) it is clear that the deformation parameter for the vibrational part of the spectrum is $\tau$, while for the rotational part it is $\tau / 2$. Therefore a relation is implied between the rotational stretching and the anharmonicity corrections. Careful empirical fits are needed in order to decide if this is a restriction or an advantage of the present model. There is no a priori reason, however, that these two physically different mechanisms be described by the same parameter. A more general version of the model, allowing for these two deformation parameters to be independent of each other, might give better results.

\section{DISCUSSION}

In this paper a deformed version of the $\mathrm{O}(4)$ and $\mathrm{U}(3)$ dynamical symmetries of the vibron model for diatomic molecules has been constructed. This has been achieved by first rewriting, through the use of the concept of complementary subalgebras, the model in a more convenient form, which is subsequently deformed. The present approach unifies into a common framework the so far separate algebraic approaches to rotational and to vibrational spectra of diatomic molecules.

For the $\mathrm{O}(4)$ limit of the present model, fittings to experimental data for diatomic molecules are required. Its $\mathrm{U}(3)$ limit can be used for the description of clustering phenomena in nuclei [49], as well as for the quasimolecular description of heavy-ion resonances [50]. The present work can be extended to the study of triatomic molecules [23]. The method of complementary subalgebras can also be used in constructing [30] the deformed versions of the $U(5)$ and $O(6)$ dynamical symmetries of the interacting boson model [24] of nuclear structure. 
[1] P. P. Kulish and N. Yu. Reshetikhin, Zap. Nauchn. Semin. LOMI 101, 101 (1981).

[2] E. K. Sklyanin, Funct. Anal. Appl. 16, 262 (1982).

[3] V. G. Drinfeld, in Proceedings of the International Congress of Mathematicians, edited by A. M. Gleason (American Mathematical Society, Providence, RI, 1987), p. 798.

[4] M. Jimbo, Lett. Math. Phys. 11, 247 (1986).

[5] D. Bonatsos, P. P. Raychev, R. P. Roussev, and Yu. F. Smirnov, Chem. Phys. Lett. 175, 300 (1990).

[6] Z. Chang and H. Yan, Phys. Lett. A 154, 254 (1991).

[7] J. G. Esteve, C. Tejel, and B. E. Villaroya, J. Chem. Phys. 96, 5614 (1992).

[8] P. P. Raychev, R. P. Roussev, and Yu. F. Smirnov, J. Phys. G 16, L137 (1990).

[9] D. Bonatsos, E. N. Argyres, S. B. Drenska, P. P. Raychev, R. P. Roussev, and Yu. F. Smirnov, Phys. Lett. B 251, 477 (1990).

[10] D. Bonatsos, A. Faessler, P. P. Raychev, R. P. Roussev, and Yu. F. Smirnov, J. Phys. A 25, 3275 (1992).

[11] D. Bonatsos, S. B. Drenska, P. P. Raychev, R. P. Roussev, and Yu. F. Smirnov, J. Phys. G 17, L67 (1991).

[12] Z. Chang, H. Y. Guo, and H. Yan, Phys. Lett. A 156, 192 (1991).

[13] Z. Chang and H. Yan, Phys. Rev. A 44, 7405 (1991).

[14] Z. Chang, H. Y. Guo, and H. Yan, Commun. Theor. Phys. 17, 183 (1992).

[15] D. Bonatsos and C. Daskaloyannis, Phys. Rev. A 46, 75 (1992).

[16] D. Bonatsos and C. Daskaloyannis, Chem. Phys. Lett. 203, 150 (1993).

[17] D. Bonatsos, E. N. Argyres, and P. P. Raychev, J. Phys. A 24, L403 (1991).

[18] D. Bonatsos, P. P. Raychev, and A. Faessler, Chem. Phys. Lett. 178, 221 (1991).

[19] D. Bonatsos, C. Daskaloyannis, and K. Kokkotas, Phys. Rev. A 45, R6153 (1992).

[20] D. Bonatsos, C. Daskaloyannis, and K. Kokkotas, J. Math. Phys. 33, 2958 (1992).

[21] F. Iachello, Chem. Phys. Lett. 78, 581 (1981).

[22] F. Iachello and R. D. Levine, J. Chem. Phys. 77, 3046 (1982).

[23] O. S. van Roosmalen, F. Iachello, R. D. Levine, and A. E.
L. Dieperink, J. Chem. Phys. 79, 2515 (1983).

[24] F. Iachello and A. Arima, The Interacting Boson Model (Cambridge University Press, Cambridge, 1987).

[25] D. Bonatsos, Interacting Boson Models of Nuclear Structure (Clarendon, Oxford, 1988).

[26] J. Van der Jeugt, J. Phys. A 25, L213 (1992).

[27] Y. F. Cao and H. L. Lin, Chem. Phys. Lett. 207, 317 (1993).

[28] F. Pan, J. Phys. B 26, L47 (1993).

[29] D. Bonatsos, A. Faessler, P. P. Raychev, R. P. Roussev, and Yu. F. Smirnov, J. Phys. A 25, L267 (1992).

[30] F. Pan, International Centre for Theoretical Physics Trieste Report, 1992 (unpublished).

[31] M. Moshinsky and C. Quesne, J. Math. Phys. 11, 1631 (1970).

[32] G. Couvreur, J. Deenen, and C. Quesne, J. Math. Phys. 24, 779 (1983).

[33] C. Quesne, J. Phys. A 18, 2675 (1985).

[34] P. M. Morse, Phys. Rev. 34, 57 (1929).

[35] F. Pan and Y. F. Cao, J. Math. Phys. 29, 2384 (1988).

[36] H. Ui, Ann. Phys. (N.Y.) 49, 69 (1968).

[37] W. J. Holman III and L. C. Biedenharn, Jr., Ann. Phys. (N.Y.) 39, 1 (1966).

[38] P. P. Kulish and E. V. Damaskinsky, J. Phys. A 23, L415 (1990).

[39] H. Ui and N. Aizawa, Mod. Phys. Lett. A 5, 237 (1990).

[40] N. Aizawa, J. Phys. A 26, 1115 (1993).

[41] L. C. Biedenharn, J. Phys. A 22, L873 (1989).

[42] A. J. Macfarlane, J. Phys. A 22, 4581 (1989).

[43] T. Maekawa, J. Math. Phys. 32, 2598 (1991).

[44] Yu. F. Smirnov, V. N. Tolstoy, and Yu. I. Kharitonov, Yad. Fiz. 53, 959 (1991) [Sov. J. Nucl. Phys. 53, 593 (1991)].

[45] Yu. F. Smirnov, V. N. Tolstoy, and Yu. I. Kharitonov, Yad. Fiz. 53, 1746 (1991) [Sov. J. Nucl. Phys. 53, 1068 (1991)].

[46] N. Aizawa, J. Math. Phys. 344, 1937 (1993).

[47] R. N. Alvarez, Diploma thesis, Moscow State University, 1992.

[48] J. L. Dunham, Phys. Rev. 41, 721 (1932).

[49] J. Cseh, J. Phys. G 19, L63 (1993).

[50] Z. Chang and H. Yan, Academia Sinica Report No. ASITP-91-25, 1991 (unpublished). 